\title{
Characteristics of BRCA1/2 pathogenic germline mutations in chinese NSCLC patients and a comparison with $\mathrm{HBOC}$
}

\author{
Zheyuan Xu', Yang Wang ${ }^{1}$, Lan Wang ${ }^{2}$, Fengxian Cui ${ }^{1}$, Libin Zhang ${ }^{1}$, Jian Xiong ${ }^{1}$ and Hao Peng ${ }^{1 *}$ (D)
}

\begin{abstract}
Background and purposes: The pathogenic BRCA1/2 germline mutations contributed to Hereditary Breast and Ovarian Cancer (HBOC) susceptibility. The features of BRCA1/2 germline mutations in non-small cell lung cancer (NSCLC) have not been systematically studied. Here we performed the first study investigating the characteristics of pathogenic BRCA1/2 germline mutations in Chinese NSCLC patients and compared them with those from Chinese HBOC.

Methods: Information on BRCA1/2 germline mutations from 9010 Chinese NSCLC patients were collected from available studies and analyzed, and compared with the BRCA1/2 germline mutations from Chinese HBOC BRCA1/2 database (LOVD database, 20,523 patients).

Results: 19 (20 carriers, $0.22 \%$ ) pathogenic BRCA1 and 60 (66 carriers, $0.73 \%$ ) pathogenic BRCA2 germline mutations from NSCLC were identified. The carrier frequency of BRCA1/2 in Chinese NSCLC patients $(86 / 9010=$ $0.95 \%)$ was significantly lower than that in Chinese breast and ovary cancer patients $(1481 / 20,523=7.2 \%)(P<$ 0.001). We found that frameshift and nonsense mutations were the predominant types of BRCA1/2 mutation in NSCLC, with no obvious hot spot mutations. No significant difference in the ratio of frameshift and nonsense mutations was found between BRCA1 and BRCA2 in NSCLC. 5 out of 19 mutations in BRCA1 and 23 out of 60 mutations in BRCA2 were novel mutations found in NSCLC that have never been reported in Chinese HBOC. A trend of higher percentage of BRCA1 nonsense mutations in the carriers was revealed in NSCLC compared with $\mathrm{HBOC}$, while no such difference was found in BRCA2 in all types of mutations.
\end{abstract}

Conclusions: BRCA1/2 germline mutations from NSCLC exhibited distinct characteristics compared with those from $\mathrm{HBOC}$ in Chinese population, including lower carrier frequency than $\mathrm{HBOC}$, higher ratio of nonsense mutations and carriers than $\mathrm{HBOC}$, and novel BRCA1/2 germline mutations never found in HBOC.

Keywords: Lung cancer, HBOC, BRCA1, BRCA2, Germline, Frameshift, Nonsense

\footnotetext{
*Correspondence: phao9375@163.com

'Department of Thoracic Surgery, The First People's Hospital of Yunnan

Province, the Affiliated Hospital of Kunming University of Science and

Technology, No. 157 Jinbi Road, 650032 Kunming, Yunnan, China

Full list of author information is available at the end of the article
}

C C The Author(s). 2021 Open Access This article is licensed under a Creative Commons Attribution 4.0 International License, which permits use, sharing, adaptation, distribution and reproduction in any medium or format, as long as you give appropriate credit to the original author(s) and the source, provide a link to the Creative Commons licence, and indicate if changes were made. The images or other third party material in this article are included in the article's Creative Commons licence, unless indicated otherwise in a credit line to the material. If material is not included in the article's Creative Commons licence and your intended use is not permitted by statutory regulation or exceeds the permitted use, you will need to obtain permission directly from the copyright holder. To view a copy of this licence, visit http://creativecommons.org/licenses/by/4.0/ The Creative Commons Public Domain Dedication waiver (http://creativecommons.org/publicdomain/zero/1.0/) applies to the data made available in this article, unless otherwise stated in a credit line to the data. 


\section{Introduction}

The germline mutations of BRCA1/2 have been comprehensively investigated in Hereditary Breast and Ovarian Cancer (HBOC). The interpretation of pathogenicity of BRCA1/2 germline mutations is based on the American College of Medical Genetics (ACMG) guidelines and provides strong tools for new mutation identification and interpretation [1]. In terms of pathogenicity, most BRCA $1 / 2$ mutations are sporadic mutations with no pathogenic nature, and these mutations are classified as benign, likely benign or VUS (variants of uncertain significance) [1]. Only pathogenic and likely pathogenic mutations are actionable and patients with these mutations need to be treated actively. PARP inhibitors have been widely used for the treatment of HBOC at first and multiple line levels with good response and compliance [2].

Apart from $\mathrm{HBOC}$, the germline BRCA1/2 mutations have also been found in a variety of other cancers, including pancreatic cancer [3], lung cancer [4], urothelial carcinoma [5] and esophageal cancer [6], etc. It is reasonable to expect that PARPi may also be effective for these patients. However, the evidence for the effectiveness of PARPi treatment on these cancers is limited so far. This is because the incidence of BRCA $1 / 2$ germline mutations in these cancers is much lower than that in HBOC [7], making it difficult to perform large-scale clinical trials. In this study, we focused on the characteristics of BRCA1/2 germline mutations in NSCLC, and compared the features of these mutations with those found in HBOC. We identified distinct mutational characteristics and novel germline mutations of BRCA1/2 in NSCLC, providing useful information for potential PARPi treatment of NSCLC with such mutations.

\section{Methods and materials}

Data collection, analysis and interpretation of BRCA1/2 mutations in NSCLC

The information on mutations and specific mutation sites were collected from three publications reporting the BRCA1/2 germline mutations in Chinese population [8-10]. Mutation sites were reorganized in protein change format and the carrier frequency was calculated for BRCA1 (Table 1) and BRCA2 (Table 2). The pathogenicity of all germline mutations were interpreted based on the guidelines for variant classification jointly issued and revised by the American College of Medical Genetics (ACMG) and the Association for Molecular Pathology (AMP) [1]. Only mutations interpreted as pathogenic were included for analysis in this study, and mutations with other pathogenicity (likely pathogenic, VUS, likely benign and benign) were excluded.

Data processing and analysis from the LOVD database

The information on mutations and specific mutation sites was downloaded from the LOVD database following the link below:

Table 1 The details of germline BRCA1 mutations and the number of carriers for lung cancer patients

\begin{tabular}{|c|c|c|c|}
\hline Mutations & Mutation Type & Number of carriers in Lung Cancer & Number of carriers in Breast and Ovary Cancer \\
\hline $11824 \mathrm{Dfs} * 3$ & Frameshift & 2 & 134 \\
\hline Exon4-splice & Splice site variant & 1 & 13 \\
\hline R1443* & Nonsense & 1 & 11 \\
\hline E489* & Nonsense & 1 & 9 \\
\hline E1158* & Nonsense & 1 & 8 \\
\hline L502Afs*2 & Frameshift & 1 & 4 \\
\hline $\mathrm{E} 720^{*}$ & Nonsense & 1 & 3 \\
\hline S1503* & Nonsense & 1 & 2 \\
\hline Exon3-splice & Splice site variant & 1 & 1 \\
\hline $\mathrm{H} 318 \mathrm{LfS}^{*} 24$ & Frameshift & 1 & 1 \\
\hline T715fs & Frameshift & 1 & 1 \\
\hline L1209* & Nonsense & 1 & 1 \\
\hline F1571fs & Frameshift & 1 & 1 \\
\hline G1743fs & Frameshift & 1 & 1 \\
\hline K339Rfs*2 & Frameshift & 1 & 0 \\
\hline E755fs & Frameshift & 1 & 0 \\
\hline Q934* & Nonsense & 1 & 0 \\
\hline K1079* & Nonsense & 1 & 0 \\
\hline Y1666* & Nonsense & 1 & 0 \\
\hline
\end{tabular}


Table 2 The details of germline BRCA2 mutations and the number of carriers for lung cancer patients

\begin{tabular}{|c|c|c|c|}
\hline Mutations & Mutation Type & Number of carriers in Lung Cancer & Number of carriers in Breast and Ovary Cancer \\
\hline Q1037* & Nonsense & 3 & 48 \\
\hline S1722fs & Frameshift & 2 & 25 \\
\hline K936fs & Frameshift & 1 & 16 \\
\hline F2801fs & Frameshift & 1 & 16 \\
\hline K157Sfs $* 24$ & Frameshift & 1 & 12 \\
\hline A938fs & Frameshift & 1 & 12 \\
\hline N3024fs & Frameshift & 1 & 10 \\
\hline M815fs & Frameshift & 1 & 7 \\
\hline S1900* & Nonsense & 1 & 7 \\
\hline N433fs & Frameshift & 1 & 6 \\
\hline R2336L & Missense & 2 & 6 \\
\hline N2135fs & Frameshift & 1 & 5 \\
\hline T3033fs & Frameshift & 1 & 5 \\
\hline S2120* & Nonsense & 1 & 4 \\
\hline R2318* & Nonsense & 1 & 4 \\
\hline $1591 \mathrm{fs}$ & Frameshift & 1 & 3 \\
\hline Q1987* & Nonsense & 1 & 3 \\
\hline Exon24-splice & Splice site variant & 1 & 3 \\
\hline $1605 f s$ & Frameshift & 1 & 2 \\
\hline $\mathrm{S} 780^{*}$ & Nonsense & 1 & 2 \\
\hline L929* & Nonsense & 1 & 2 \\
\hline Y949fs & Frameshift & 1 & 2 \\
\hline M2235fs & Frameshift & 1 & 2 \\
\hline S2984* & Nonsense & 1 & 2 \\
\hline G3134Afs*29 & Frameshift & 1 & 2 \\
\hline D252fs & Frameshift & 2 & 1 \\
\hline P1145* & Nonsense & 1 & 1 \\
\hline L1908fs & Frameshift & 1 & 1 \\
\hline N2137fs & Frameshift & 1 & 1 \\
\hline Exon16-splice & Splice site variant & 2 & 1 \\
\hline Q2530* & Nonsense & 1 & 1 \\
\hline L2573* & Nonsense & 1 & 1 \\
\hline exon21-splice & Splice site variant & 1 & 1 \\
\hline $8487+1 G>C$ & Splice site variant & 1 & 1 \\
\hline K3004fs & Frameshift & 1 & 1 \\
\hline R3052W & Missense & 1 & 1 \\
\hline M3118fs & Frameshift & 1 & 1 \\
\hline $\mathrm{S} 158^{*}$ & Nonsense & 1 & 0 \\
\hline D306fs & Frameshift & 1 & 0 \\
\hline E357* & Nonsense & 1 & 0 \\
\hline T598fs & Frameshift & 1 & 0 \\
\hline E790* & Nonsense & 1 & 0 \\
\hline Y828* & Nonsense & 1 & 0 \\
\hline N1055fs & Frameshift & 1 & 0 \\
\hline
\end{tabular}


Table 2 The details of germline BRCA2 mutations and the number of carriers for lung cancer patients (Continued)

\begin{tabular}{llll}
\hline Mutations & Mutation Type & Number of carriers in Lung Cancer & Number of carriers in Breast and Ovary Cancer \\
\hline D1210fs & Frameshift & 1 & 0 \\
K1239fs & Frameshift & 1 & 0 \\
D1476fs & Frameshift & 1 & 0 \\
I1485Nfs*3 & Frameshift & 1 & 0 \\
L1616fs & Frameshift & 1 & 0 \\
E1625fs & Frameshift & 1 & 0 \\
E1646Nfs*19 & Frameshift & 1 & 0 \\
Y1661* & Nonsense & 1 & 0 \\
V1681fs & Frameshift & 1 & 0 \\
R1694* & nonsense & 1 & 0 \\
K1964* & Nonsense & 1 & 0 \\
H1966fs & Frameshift & 1 & 0 \\
12149fs & Frameshift & 1 & 0 \\
G2274fs & Frameshift & 1 & 0 \\
R2896fs & Frameshift & 1 & 0 \\
E3258fs & Frameshift & 1 & 0 \\
\hline
\end{tabular}

https://databases.lovd.nl/shared/variants?search owned_by_=\%3D\%22Xianqi $\% 20 \mathrm{Gao} \% 22$.

The set of data was reported in a recent publication comprehensively reviewed and summarized the BRCA1/2 germline mutations from HBOC in Chinese population from 1999 to 2017 [11]. Therefore, the database represents a full collection of Chinese BRCA1/2 germline mutations so far. The BRCA1/2 mutations found in NSCLC were searched in the LOVD database, and the corresponding carrier frequency in $\mathrm{HBOC}$ was determined and compared with that from NSCLC (Tables 1 and 2). The mutational frequency, carrier frequency, and mutational type information for HBOC was also collected and summarized for analysis.

\section{Statistics and figure plot}

Statistical analysis was performed, and figures were plotted with GraphPad Prism 5.0 software (GraphPad Software, Inc, La Jolla, CA 92,037, USA) or the R software (https://www.r-project.org/). Chi-square test and Fisher test were performed when rate or percentage was compared for significance. $\mathrm{P}<0.05$ was regarded as significant difference.

\section{Results}

Main features of the pathogenic BRCA1/2 germline mutations in NSCLC patients

The information for all pathogenic BRCA1/2 mutations found in NSCLC was collected and analyzed. All mutations were plotted in a scheme to show the distribution

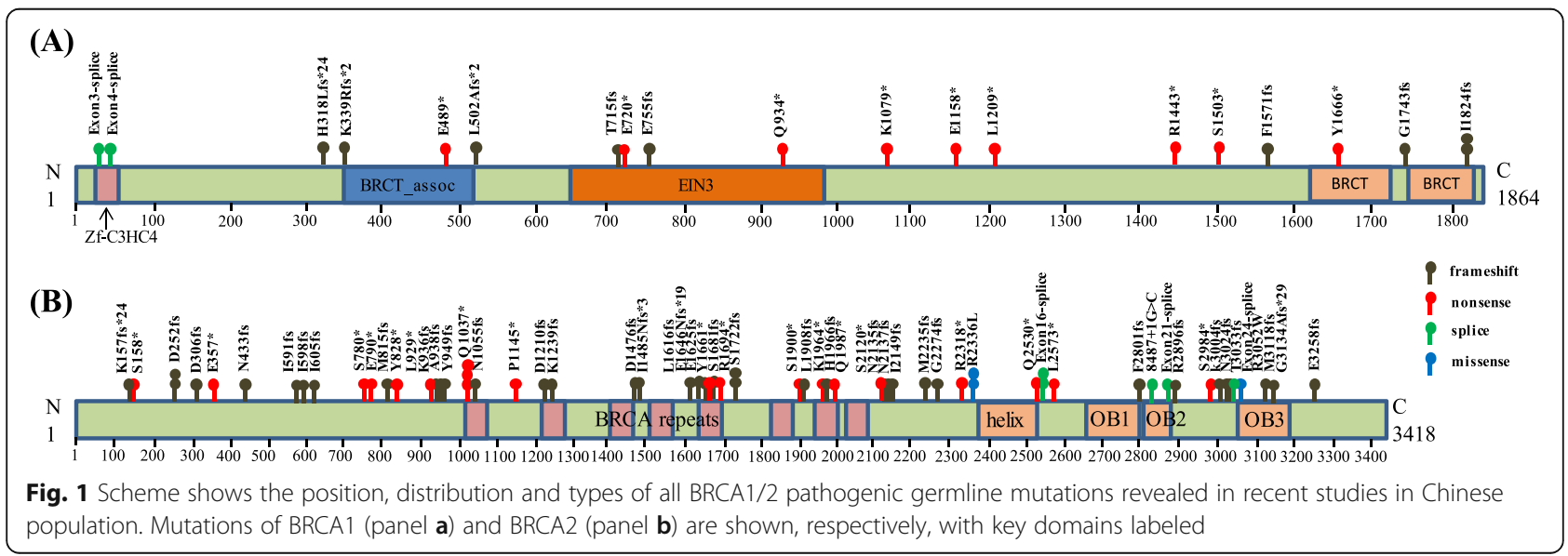


and types of mutations (Fig. 1). 19 (20 carriers) pathogenic BRCA1 (Fig. 1A) and 60 (66 carriers) pathogenic BRCA2 (Fig. 1B) germline mutations were identified. It can be observed that frameshift and nonsense mutations were the predominant types of BRCA1/2 mutation in NSCLC. 8 out 19 mutations were frameshift and 9 out of 19 mutations were nonsense in BRCA1 (Table 1). 36 out of 60 mutations were frameshift and 18 out of 60 mutations were nonsense in BRCA2 (Table 2). No significant difference in the ratio of frameshift and nonsense mutations was found between BRCA1 and BRCA2 in NSCLC. The distribution of the mutations appeared to be sporadic in both BRCA1 and BRCA2, and mutations can be found inside and outside the key functional domains, and there were no obvious hot spot mutations. The percentage of BRCA1/2 germline mutation carriers in Chinese NSCLC patients $(86 / 9010=0.95 \%)$ was significantly lower than that in Chinese breast and ovary cancer patients $(1481 / 20,523=7.2 \%) \quad(\mathrm{P}<0.001)$, suggesting a cancer-type dependent incidence of BRCA1/2 germline mutations.

\section{Comparison of BRCA1/2 germline mutation characteristics} between NSCLC and HBOC

BRCA1/2 mutational information of HBOC from the LOVD database was summarized and the mutational features were compared with those from NSCLC. The number of variants or carriers and the percentage of BRCA1/2 in NSCLC and HBOC are shown in Fig. 2. Potential higher percentage of BRCA1 nonsense mutations and the carriers was revealed in NSCLC (Fig. 2A) compared with $\mathrm{HBOC}$ (Fig. 2B) (Chi-square test, $\mathrm{P}=0.069$ for variant percentage and $\mathrm{P}=0.060$ for carrier percentage). In contrast, no such difference was found in BRCA2 in all types of mutations between NSCLC (Fig. $2 \mathrm{C}$ ) and HBOC (Fig. 2D).

The individual mutations found in NSCLC were compared with those in HBOC (Fig. 3). It was revealed that 5 out of 19 pathogenic mutations in BRCA1 (Fig. 3A; Table 1) and 23 out of 60 pathogenic mutations in BRCA2 (Fig. 3B; Table 2) were novel mutations found in NSCLC that have never been reported in Chinese HBOC. These novel mutations were all low-frequency mutations in NSCLC, suggesting their sporadic nature.

\section{Discussion}

The BRCA1/2 germline mutations in HBOC have been widely studied in many reports, and databases such as BIC, ClinVar, BRCA Share and ENIGMA, collected huge number of BRCA1/2 germline mutations [11, 12]. However, the mutations recorded by these databases are mainly from Caucasian, black and Jewish populations. It was not until very recently that the database for BRCA1/ 2 germline mutations in Chinese population was established [11]. Studies so far found that BRCA1/2 germline mutations varied substantially across different populations. Unlike other hereditary cancer syndrome such as Lynch syndrome, no definite hot spot BRCA1/2 mutations were found in several populations. However, definite hot spot mutations were observed in Ashkenazi Jewish population, exhibiting clear founder effect [11]. Reports in Chinese population did not find founder effect, although some high frequency mutations were observed [11]. This could be due to the fact that Jewish

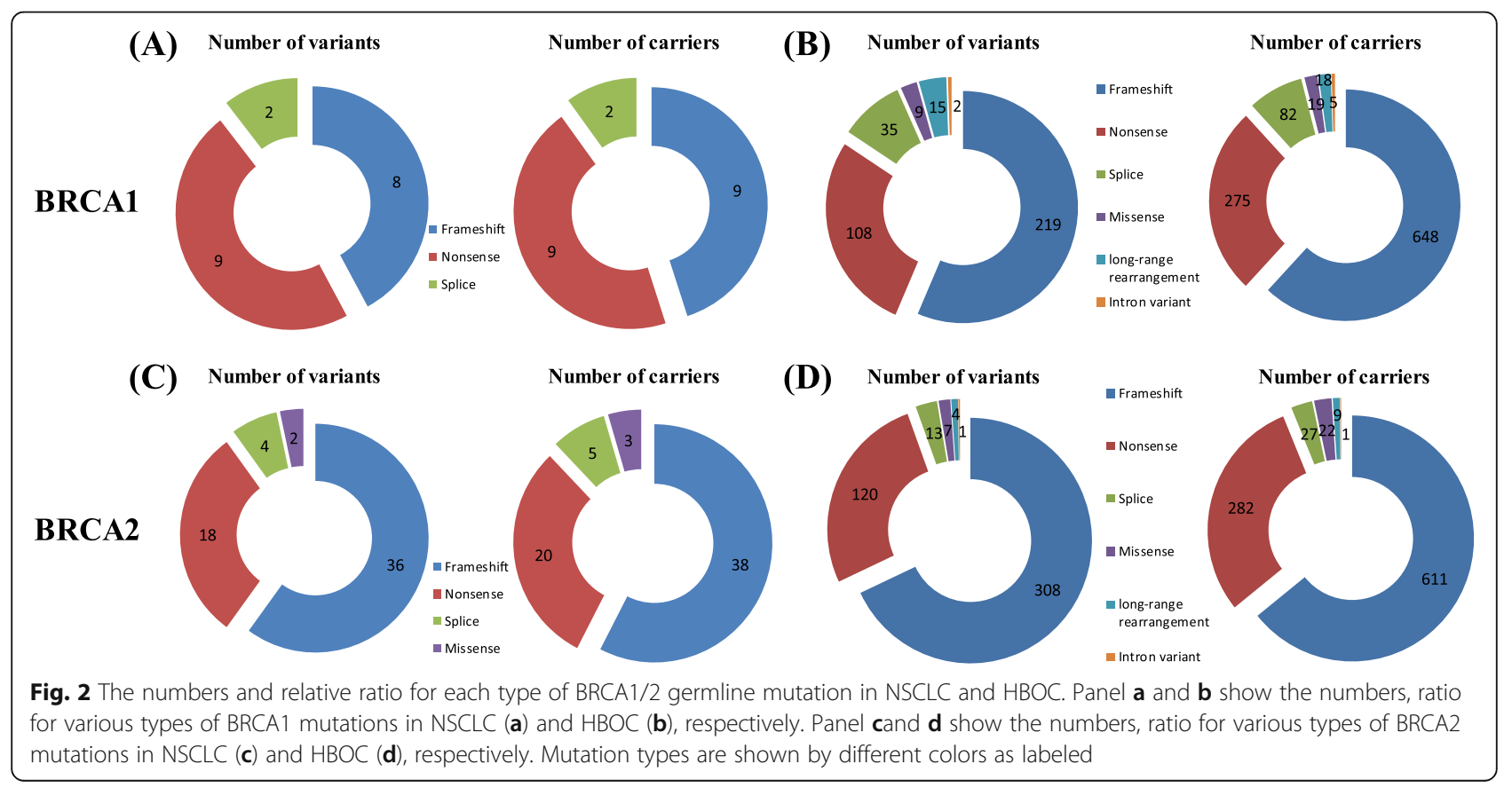




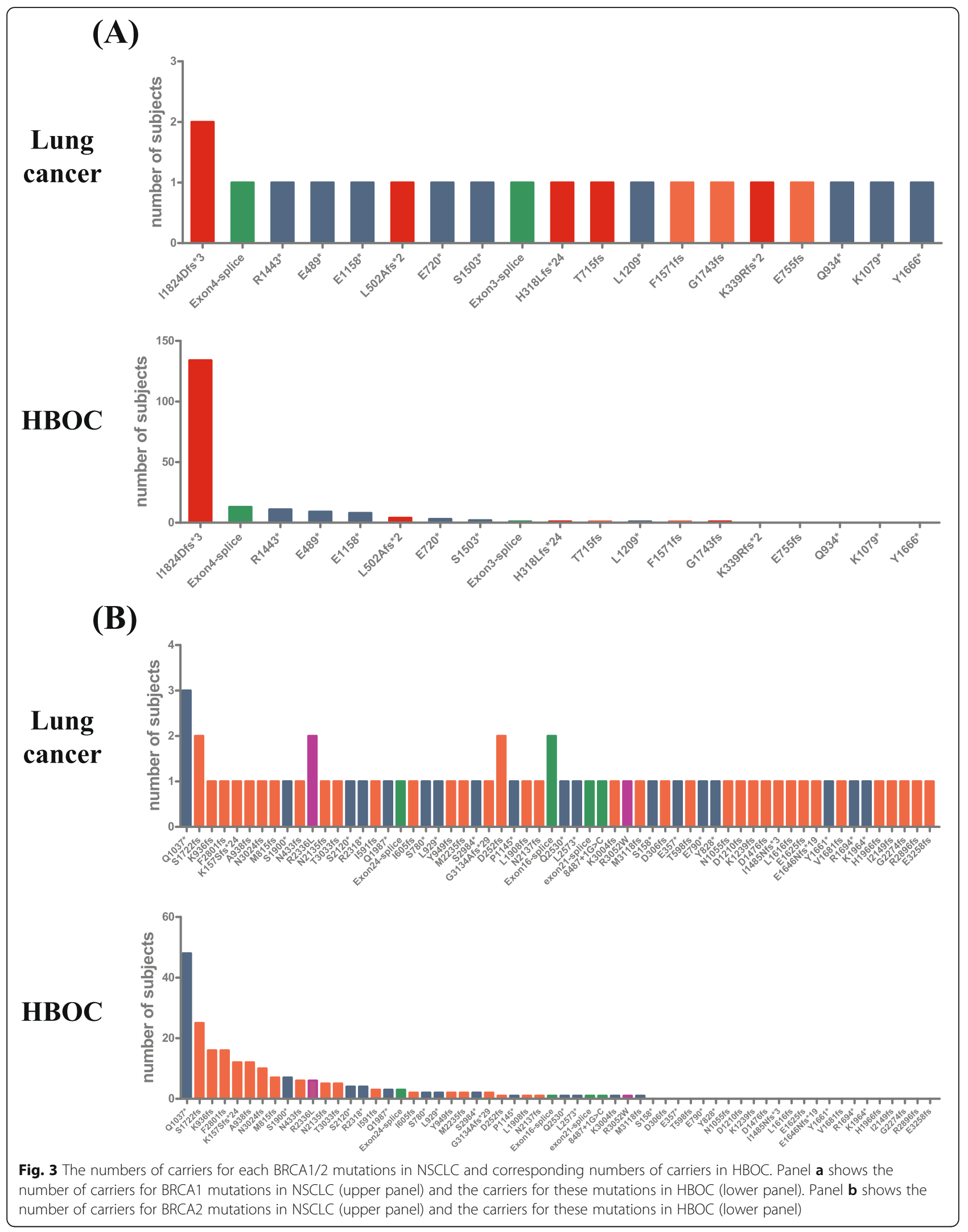


population had relatively strict endogamy while largescale population migration across east and central Asia happened in Chinese population Therefore, studies on BRCA1/2 germline mutations must consider the discrepancy across different races. The panorama of BRCA1/2 mutations in Chinese was recently established to facilitate the diagnosis and therapy in the population.

BRCA1/2 germline mutations were not only reported in $\mathrm{HBOC}$, but also reported in many other cancer types, including pancreatic cancer [3], lung cancer [4], urothelial carcinoma [5] and esophageal cancer [6]. The frequency of BRCA $1 / 2$ germline mutations in these cancer appeared to be significantly lower than that in HBOC, showing clear HBOC preference. For example, a study involving 854 patients with pancreatic ductal adenocarcinoma showed that 12 patients (1.4\%) had BRCA2 and 3 patients $(0.35 \%)$ had BRCA1 deleterious germline mutations [13]. Our study on Chinese NSCLC patients found 20 patients $(0.22 \%)$ with BRCA1 and 66 patients $(0.73 \%)$ with BRCA2 pathogenic germline mutations, representing a comprehensive report on the incidence of BRCA1/2 germline mutations in Chinese NSCLC. This incidence was much lower than that reported in Chinese HBOC, in which $5.74 \%$ of breast cancer and $21.79 \%$ of ovarian cancer patients had BRCA1/2 germline mutations, with an overall pathogenic mutation frequency of $69.9 \%$ and $71.1 \%$ for BRCA1 and BRCA2 exons, respectively [11]. Indeed, studies have suggested that no significant difference was found in the ratio of NSCLC patients with smoking history between those with or without pathogenic germline mutations $[8,10]$, and germline mutations in driver oncogenes and inherited lung cancer risk may be independent of smoking history [14]. These observations suggested that the influence of BRCA1/2 germline mutations in NSCLC may not be as big as that in $\mathrm{HBOC}$, and factors other than germline variants may play more important roles in sporadic lung cancer patients, most likely environmental factors including smoking [10].

We found that most pathogenic mutations in BRCA1/ 2 in NSCLC were frameshift and nonsense mutation. Indeed, these mutations may cause large fragment aberrancies and lead to protein dysfunction, which explains their pathogenic nature. Similar trend was also found in HBOC, in which frameshift appeared to be the mutation type with highest ratio in both BRCA1 and BRCA2 [11]. In contrast, we found that the BRCA1 nonsense mutations in NSCLC showed a trend of higher ratio than that in HBOC. If this was real, the observation suggested a preference of nonsence mutation in BRCA1 in NSCLC, however, this could be affected by the limited number of mutations in BRCA1 in NSCLC, as only a total of 20 patients were involved. No significant difference in the ratio of frameshift and nonsense mutations was found in
BRCA2 between NSCLC and HBOC, suggesting a similar mutation type distribution across different cancers. This observation also indicated that the sporadic distribution and no-hot-spot nature of BRCA1/2 mutations may exist in all cancers and possibly in general population, while the difference in BRCA1/2 incidence across cancer types may be due to the distinct enrichment effect of BRCA1/2 mutations in different cancers.

An interesting observation in this study is that family history of lung cancer has been seen in $57.14 \%(8 / 14)$ of lung cancer patients with pathogenic germline mutation, and in $32.35 \%(11 / 34)$ of patients with likely pathogenic germline mutations [10]. Therefore, it can be suggested that NSCLC did happen to a family when pathogenic germline mutations were inherited. Meanwhile, the same study also reported that multiple cancer types were found in $21.43 \%(3 / 14)$ of patients with pathogenic germline mutations, and in $11.76 \%(4 / 34)$ of patients with likely pathogenic germline mutations, suggesting that pathogenic or likely pathogenic germline mutations may also increase the risk of other cancers besides lung cancer. This was also reported in a couple of case studies. Boettger et al. found a lung cancer patient with BRCA2 pathogenic germline mutation with strong family history of breast cancer [15], and Marafie et al. also reported a lung cancer patient from an extended family segregating different types of hereditary cancer over several generations, including lung, breast, ovarian, colon, prostate and renal cancers [16]. Therefore, it can be suggested that the emergence of cancers in these families was not due to the co-finding of pathogenic germline mutations with environmental factor-relevant cancers but was correlated with the risk from germline mutations.

In this study, we also identified several novel pathogenic germline mutations in NSCLC that have never been found in Chinese HBOC patients. New germline mutations of BRCA $1 / 2$ have been emerging all the time in the past 20 years in $\mathrm{HBOC}$ [11]. It appeared that the sporadic nature of the BRCA1/2 mutations may continuously generate novel mutations, not only in $\mathrm{HBOC}$, but also in NSCLC. Therefore, the novel mutations found in NSCLC may not be cancer-specific, but related to population-based sporadic distribution. If the examined population is large enough, more novel mutations may be revealed. It was interesting in this study that a similar trend of mutational frequency may exist between NSCL $\mathrm{C}$ and HBOC. For example, BRCA2 Q1037* was the mutation with highest frequency in both NSCLC and HBOC. Although the number of patients was limited in NSCLC, this trend could be a reflection of mutational spectrum similarity in Chinese population across different cancers. Therefore, we speculate that the background BRCA1/2 mutational status in cancers may be 
similar, and the emergence of certain mutations in a cancer may be random, while the enrichment of the mutations may be cancer-specific.

PARP inhibitors (PARPi) have been widely used in the treatment of HBOC, and their effectiveness have been proved by many studies at first-line and multiline levels [17, 18]. Maintenance therapy with PARPi has been approved for HBOC therapy in patients who are sensitive to platinumbased chemotherapy, which plays a key role and has been applied in the first-line therapy of ovarian and breast cancers for more than two decades [19]. Although various combinations with platinum-based reagents have been investigated over the years to find the best strategy for optimal response and minimal toxicity, it seems difficult to further improve the response while balance the toxicity of these drugs [2022]. Maintenance therapy was therefore developed to solve the issue in patients who achieved an initial response to chemotherapy with the goal to maintain the response with an acceptable toxicity [23]. Therefore, platinum-based chemotherapy is still important for initial therapy of these patients, and PARPi has crucial roles in maintaining the response and reducing the side effects.

Advances have also been made in the application of PARPi in cancers other than HBOC with BRCA1/2 germline or somatic mutations. For example, for metastatic pancreatic cancer patients with germline BRCA mutations who had not progressed during first-line platinum-based chemotherapy, progression-free survival was longer with maintenance Olaparib than with placebo [24]. There are also a couple of ongoing clinical trials designed to evaluate the safety and efficacy of PARPi in urothelial carcinoma as monotherapy or in combination with other drugs, including two ongoing phase II clinical trials (NCTXXXX and NCTXXXX), but specific efficacy data have not been reported [25]. In contrast, no clinical trial data is available so far for NSCLC treatment with PARPi, possibly due to the plenty of therapeutic methods for NSCLC and the very low incidence of BRCA1/2 germline mutations in NSCLC.

In conclusion, our study systematically investigated the characteristics of BRCA1/2 germline mutations in Chinese NSCLC patients, and compared the features of the mutations with those from Chinese HBOC. We found that BRCA1/2 germline mutations in NSCLC had lower carrier frequency than $\mathrm{HBOC}$, potential higher ratio of BRCA1 nonsense mutations and carriers than HBOC, and revealed several novel BRCA1/2 germline mutations that have never been reported in Chinese HBOC.

\section{Acknowledgements}

Not applicable.

\section{Authors' contributions}

The study was designed by Zheyuan Xu, Hao Peng and Jian Xiong. Data for lung cancer BRCA1/2 germline mutations was collected by Zheyuan Xu. Data for HBOC BRCA1/2 germline mutations was collected by Zheyuan $\mathrm{Xu}$, Yang
Wang, Lan Wang, Fengxian Cui and Libin Zhang. Data was analyzed by Zheyuan Xu, Yang Wang, Lan Wang, Fengxian Cui and Libin Zhang. Figures were made by Zheyuan $\mathrm{Xu}$ and Yang Wang. The manuscript was written by Zheyuan Xu and proof read by Hao Peng. The paper was submitted by Hao Peng. The authors read and approved the final manuscript.

\section{Funding}

This study was supported by the Yunnan Clinical Medicine Center for Respiratory Diseases (Grant Number 2019LCZXKF-HX03), Science and Technology Department of Yunnan Province (Grant Number 2019 FE001 (-115)), and Yunnan province medical science and technology project (Grant Number 2018NS0271).

\section{Availability of data and materials}

The data that support the findings of this study are openly available in LOVD at https://databases.lovd.nl/shared/variants?search_owned_by_=\%3D\%22 Xianqi\%20Gao\%22. Other data are available from the corresponding author upon reasonable request.

\section{Ethics approval and consent to participate}

This article is a database research and therefore ethics approval and consent to participate are not needed.

\section{Consent for publication}

This article is a database research and therefore consent for publication is not needed.

\section{Competing interests}

The authors claim no competing interests for this study.

\section{Author details}

'Department of Thoracic Surgery, The First People's Hospital of Yunnan Province, the Affiliated Hospital of Kunming University of Science and Technology, No. 157 Jinbi Road, 650032 Kunming, Yunnan, China. ${ }^{2}$ Department of Anesthesiology, The First People's Hospital of Yunnan Province, The Affiliated Hospital of Kunming University of Science and Technology, No. 157 Jinbi Road, 650032 Kunming, Yunnan, China.

Received: 2 September 2020 Accepted: 29 January 2021

Published online: 09 February 2021

\section{References}

1. Richards S, Aziz N, Bale S, et al. Standards and guidelines for the interpretation of sequence variants: a joint consensus recommendation of the American College of Medical Genetics and Genomics and the Association for Molecular Pathology. Genet Med. 2015;17:405-24.

2. Yamauchi $\mathrm{H}$, Takei J. Management of hereditary breast and ovarian cancer. Int J Clin Oncol. 2018;23(1):45-51.

3. Grant RC, Selander I, Connor AA, et al. Prevalence of germline mutations in cancer predisposition genes in patients with pancreatic cancer. Gastroenterology. 2015;148(3):556-64

4. Parry EM, Gable DL, Stanley SE, et al. Germline Mutations in DNA repair genes in lung adenocarcinoma. J Thorac Oncol. 2017;12(11):1673-8.

5. Nassar AH, Abou Alaiwi S, AlDubayan SH, et al. Prevalence of pathogenic germline cancer risk variants in high-risk urothelial carcinoma. Genet Med. 2020;22(4):709-18.

6. Ko JM, Ning L, Zhao XK, et al. BRCA2 loss-of-function germline mutations are associated with esophageal squamous cell carcinoma risk in Chinese. Int J Cancer. 2020;146(4):1042-51.

7. Friebel TM, Domchek SM, Rebbeck TR. Modifiers of cancer risk in BRCA1 and BRCA2 mutation carriers: systematic review and meta-analysis. J Natl Cancer Inst. 2014;106(6):dju091.

8. Hu X, Yang D, Li Y, et al. Prevalence and clinical significance of pathogenic germline BRCA1/2 mutations in Chinese non-small cell lung cancer patients. Cancer Biol Med. 2019;16(3):556-64.

9. Tian $P$, Cheng $X$, Zhao $Z$, et al. Spectrum of pathogenic germline mutations in Chinese lung cancer patients through next-generation sequencing. Pathol Oncol Res. 2020:26(1):109-14.

10. Liu M, Liu X, Suo P, et al. The contribution of hereditary cancer-related germline mutations to lung cancer susceptibility. Transl Lung Cancer Res. 2020;9(3):646-58. 
11. Gao X, Nan X, Liu Y, et al. Comprehensive profiling of BRCA1 and BRCA2 variants in breast and ovarian cancer in Chinese patients. Hum Mutat. 2020; 41(3):696-708.

12. Cline MS, Liao RG, Parsons MT, et al. BRCA Challenge: BRCA Exchange as a global resource for variants in BRCA1 and BRCA2. PLoS Genet. 2018;14(12): e1007752.

13. Shindo $\mathrm{K}, \mathrm{Yu} J$, Suenaga $\mathrm{M}$, et al. Deleterious germline mutations in patients with apparently sporadic pancreatic adenocarcinoma. J Clin Oncol. 2017; 35(30):3382-90.

14. Oxnard GR, Nguyen KS, Costa DB. Germline mutations in driver oncogenes and inherited lung cancer risk independent of smoking history. J Nat Cancer Inst. 2014;106(1):djt361.

15. Boettger MB, Sergi C, Meyer P. BRCA1/2 mutation screening and LOH analysis of lung adenocarcinoma tissue in a multiple-cancer patient with a strong family history of breast cancer. J Carcinog. 2003:2(1):5. Published 2003 Oct 2

16. Marafie MJ, Dashti M, Al-Mulla F. Identification of a rare germline NBN gene mutation by whole exome sequencing in a lung-cancer survivor from a large family with various types of cancer. Fam Cancer. 2017;16(3):389-94.

17. Mirza MR, Coleman RL, González-Martín A, et al. The forefront of ovarian cancer therapy: update on PARP inhibitors. Ann Oncol. 2020;50923-7534(20): 39891-4.

18. Jerez Y, Márquez-Rodas I, Aparicio I, Alva M, Martín M, López-Tarruella S. Poly (ADP-ribose) polymerase inhibition in patients with breast cancer and BRCA 1 and 2 mutations. Drugs. 2020;80(2):131-46.

19. Markman M. Pharmaceutical management of ovarian cancer: current status. Drugs. 2019;79(11):1231-9. https://doi.org/10.1007/s40265-019-01158-1.

20. McGuire WP, Hoskins WJ, Brady MF, et al. Cyclophosphamide and cisplatin compared with paclitaxel and cisplatin in patients with stage III and stage IV ovarian cancer. N Engl J Med. 1996;334(1):1-6.

21. Ozols RF, Bundy BN, Greer BE, et al. Phase III trial of carboplatin and paclitaxel compared with cisplatin and paclitaxel in patients with optimally resected stage III ovarian cancer: a Gynecologic Oncology Group study. J Clin Oncol. 2003;21(17):3194-200.

22. Vasey PA, Jayson GC, Gordon A, et al. Phase III randomized trial of docetaxel-carboplatin versus paclitaxel-carboplatin as first-line chemotherapy for ovarian carcinoma. J Natl Cancer Inst. 2004;96(22):168291.

23. Heo YA, Dhillon S. Olaparib tablet: A review in ovarian cancer maintenance therapy. Target Oncol. 2018;13(6):801-8. https://doi.org/10.1007/s11523-0180606-X.

24. Golan T, Hammel P, Reni M, et al. Maintenance Olaparib for germline BRCAMutated metastatic pancreatic cancer. N Engl J Med. 2019;381(4):317-27.

25. Garje R, Vaddepally RK, Zakharia Y. PARP inhibitors in prostate and urothelial cancers. Front Oncol. 2020;10:114.

\section{Publisher's Note}

Springer Nature remains neutral with regard to jurisdictional claims in published maps and institutional affiliations.

Ready to submit your research? Choose BMC and benefit from:

- fast, convenient online submission

- thorough peer review by experienced researchers in your field

- rapid publication on acceptance

- support for research data, including large and complex data types

- gold Open Access which fosters wider collaboration and increased citations

- maximum visibility for your research: over $100 \mathrm{M}$ website views per year

At $\mathrm{BMC}$, research is always in progress.

Learn more biomedcentral.com/submissions 